\title{
A Robust Exponentially Weighted Moving Average Control Chart for the Process Mean
}

\author{
Michael B. C. Khoo \\ Universiti Sains, Malaysia, mkbc@usm.my \\ S. Y. Sim \\ Universiti Sains Malaysia
}

Follow this and additional works at: http:// digitalcommons.wayne.edu/jmasm

Part of the Applied Statistics Commons, Social and Behavioral Sciences Commons, and the $\underline{\text { Statistical Theory Commons }}$

\section{Recommended Citation}

Khoo, Michael B. C. and Sim, S. Y. (2005) "A Robust Exponentially Weighted Moving Average Control Chart for the Process Mean," Journal of Modern Applied Statistical Methods: Vol. 5 : Iss. 2 , Article 21.

DOI: $10.22237 /$ jmasm/1162354800

Available at: http://digitalcommons.wayne.edu/jmasm/vol5/iss2/21

This Regular Article is brought to you for free and open access by the Open Access Journals at DigitalCommons@WayneState. It has been accepted for inclusion in Journal of Modern Applied Statistical Methods by an authorized editor of DigitalCommons@WayneState. 


\title{
A Robust Exponentially Weighted Moving Average Control Chart for the Process Mean
}

\author{
Michael B.C. Khoo S.Y. Sim \\ School of Mathematical Sciences, \\ Universiti Sains Malaysia
}

To date, numerous extensions of the exponentially weighted moving average, EWMA charts have been made. A new robust EWMA chart for the process mean is proposed. It enables easier detection of outliers and increase sensitivity to other forms of out-of-control situation when outliers are present.

Key words: Exponentially weighted moving average (EWMA), cumulative sum (CUSUM), Shewhart, process mean, sample mean, sample range, average run length (ARL)

\section{Introduction}

The EWMA chart is a good alternative to the Shewhart chart in the detection of small shifts. The EWMA chart constructed from the sample mean is first developed by Roberts (1959). Since then various extensions of the EWMA charts have been proposed. Sweet (1986) proposed two models to construct simultaneous control charts to monitor the mean and the variance of a process using the EWMA. Crowder (1987 \& 1989) provided average run length (ARL) tables and graphs for the selection of the optimum values of the EWMA control chart parameters in the design of an EWMA chart. $\mathrm{Ng} \&$ Case (1989) presented several EWMA control chart schemes based on individual measurement,

Michael B. C. Khoo is a member of the American Society for Quality (ASQ) and the American Statistical Association (ASA). He also serves as a member of the editorial boards of Quality Engineering, Quality Management Journal and Journal of Modern Applied Statistical Methods. His research interest is in statistical process control. S. Y. Sim graduated from the School of Mathematical Sciences, Universiti Sains Malaysia with an M.Sc. degree. sample mean, sample range and moving range statistics. Lucas \& Saccucci (1990) showed that a fast initial response (FIR) feature is useful for the EWMA chart, especially for small values of smoothing constants. Rhoads, Montgomery \& Mastrangelo (1996) proposed a scheme which is superior to that of Lucas \& Saccucci (1990). MacGregor \& Harris (1993) suggested an approach of using the EWMA based statistics in the monitoring of the process standard deviation. Gan (1990) proposed three modified EWMA charts for the Poisson data. A better procedure for using the EWMA chart for Poisson count is given by Borror, Champ \& Rigdon (1998). Somerville, Montgomery \& Runger (2002) developed a smoothing and filtering method using the EWMA and Poisson probabilities which separates the two distributions in a particle count data stream into a base process and an outlier process followed by applying statistical monitoring schemes to each of them. A Bernoulli EWMA is suggested to monitor the outlier process.

The EWMA control chart scheme for the sample mean proposed by Ng \& Case (1989) is constructed by assuming that the data used in the computation of the limits are outlier free. This assumption may not be true in real situations since outliers often occur in the data used to compute the control limits. Outliers may consist of single unusual values which happen due to a sporadic special cause. Such outliers act only on occasional observations in a subgroup 
and not on subgroups as a whole. These outliers have to be detected, investigated and the special cause removed if possible. The presence of outliers will reduce the sensitivity of a control chart because the control limits are stretched so that the detection of the outliers themselves become more difficult. Furthermore, these stretched limits also make it more difficult for other types of out-of-control signals to be detected (Rocke, 1989, 1992). The purpose of this article is to propose a robust EWMA (EWMASMQ) chart for the process mean as an alternative which is superior to the standard EWMA (EWMASM) chart for the process mean. The EWMASMQ chart is constructed based on the limits that are set using an estimate of the process standard deviation using the average of the subgroup interquartile ranges (IQRs) rather than the average of the subgroup ranges in the case of the EWMASM. Thus, the EWMASMQ chart is less affected by outliers compared to the EWMASM chart. The next section gives an overview of the EWMASM chart.

\section{The EWMASM Chart}

The EWMASM chart is based on the following statistic (Ng \& Case, 1989):

$$
\hat{W}_{t}=\alpha \bar{X}_{t}+(1-\alpha) \hat{W}_{t-1}
$$

where $\bar{X}_{t}$ is the mean of sample $t$ and $\alpha$ is a weighting constant. In the estimation of the limits based on the process data, $m$ subgroups of size $n$ each are taken and then the average of the $m$ sample means, $\overline{\bar{X}}$ is computed. $\overline{\bar{X}}$ is used as the starting point, i.e., $\hat{W}_{0}=\overline{\bar{X}}$. The average of the $m$ sample ranges is computed to be $\bar{R}$.

The upper and lower control limits of the EWMASM chart are

$$
U C L=\overline{\bar{X}}+F_{1} \bar{R}
$$

and

$$
L C L=\overline{\bar{X}}-F_{1} \bar{R}
$$

respectively, where

$$
F_{1}=\frac{3}{d_{2} \sqrt{n}} \sqrt{\frac{\alpha}{2-\alpha}}
$$

In equation (3), $d_{2}$ is a standard constant whose value depends on the sample size $n$. The values of $d_{2}$ and $F_{1}$ ( $\mathrm{Ng} \&$ Case, 1989) for the various sample sizes $n$ are given in Tables 1 and 2 respectively.

A Proposed EWMASMQ Chart For The Process Mean

Similar to the EWMASM chart, every observed sample mean is transformed into a corresponding EWMA before it is plotted on the EWMASMQ chart. Let $\bar{X}_{t}$ represents the mean of sample $t$. Every $\bar{X}_{t}$ will be transformed into a corresponding EWMA, $\hat{W}_{t}$, using the transformation

$$
\hat{W}_{t}=\alpha \bar{X}_{t}+(1-\alpha) \hat{W}_{t-1}, t=1,2, \ldots
$$

When developing the EWMASMQ chart based on process data, $m$ subgroups of size $n$ each must be taken to compute the estimate of the proces mean. The average of the $m$ sample means will be used as the starting point, i.e., $\hat{W}_{0}=\overline{\bar{X}}$. For the EWMASMQ chart, the interquartile range, IQR is defined as $X_{(b)}-X_{(a)}$, where ( ) denotes the order statistics $a=[n / 4]+1$ and $b=n-a+1$. Here, [y] represents the greatest integer that is less than or equal to $y$. It is shown by Rocke (1992) that the mathematical expectation of IQR can be defined as

$$
E(\mathrm{IQR})=d_{2}^{Q} \sigma_{X}
$$


Table 1. Factors for the EWMASM and EWMASMQ Charts

\begin{tabular}{ccccc}
\hline$n$ & $a$ & $b$ & $d_{2}$ & $d_{2}^{\mathrm{Q}}$ \\
\hline 2 & 1 & 2 & 1.128 & 1.1284 \\
3 & 1 & 3 & 1.693 & 1.6926 \\
4 & 2 & 3 & 2.059 & 0.5940 \\
5 & 2 & 4 & 2.326 & 0.9900 \\
& & & & \\
6 & 2 & 5 & 2.534 & 1.2835 \\
7 & 2 & 6 & 2.704 & 1.5147 \\
8 & 3 & 6 & 2.847 & 0.9456 \\
9 & 3 & 7 & 2.970 & 1.1439 \\
10 & 3 & 8 & 3.078 & 1.3121 \\
& & & & \\
11 & 3 & 9 & 3.173 & 1.4577 \\
12 & 4 & 9 & 3.258 & 1.0737 \\
13 & 4 & 10 & 3.336 & 1.2057 \\
14 & 4 & 11 & 3.407 & 1.3235 \\
15 & 4 & 12 & 3.472 & 1.4298 \\
& & & & \\
16 & 5 & 12 & 3.532 & 1.1400 \\
17 & 5 & 13 & 3.588 & 1.2389 \\
18 & 5 & 14 & 3.640 & 1.3269 \\
19 & 5 & 15 & 3.689 & 1.4132 \\
20 & 6 & 15 & 3.735 & 1.1806 \\
\hline
\end{tabular}

Table 2. Factors of Control Limits for the EWMASM, $F_{1}$

\begin{tabular}{|c|c|c|c|c|c|c|c|c|c|c|c|}
\hline \multirow{2}{*}{$n$} & \multicolumn{11}{|c|}{$\alpha$} \\
\hline & 0 & 0.10 & 0.20 & 0.30 & 0.40 & 0.50 & 0.60 & 0.70 & 0.80 & 0.90 & 1.00 \\
\hline 2 & 0.000 & 0.431 & 0.627 & 0.790 & 0.940 & 1.085 & 1.231 & 1.380 & 1.535 & 1.701 & 1.880 \\
\hline 3 & 0.000 & 0.235 & 0.341 & 0.430 & 0.512 & 0.591 & 0.670 & 0.751 & 0.835 & 0.925 & 1.023 \\
\hline 4 & 0.000 & 0.167 & 0.243 & 0.306 & 0.365 & 0.421 & 0.477 & 0.535 & 0.595 & 0.659 & 0.729 \\
\hline 5 & 0.000 & 0.132 & 0.192 & 0.242 & 0.289 & 0.333 & 0.378 & 0.423 & 0.471 & 0.522 & 0.577 \\
\hline 6 & 0.000 & 0.111 & 0.161 & 0.203 & 0.242 & 0.279 & 0.316 & 0.354 & 0.394 & 0.437 & 0.483 \\
\hline 7 & 0.000 & 0.096 & 0.140 & 0.176 & 0.210 & 0.242 & 0.274 & 0.307 & 0.342 & 0.379 & 0.419 \\
\hline 8 & 0.000 & 0.086 & 0.124 & 0.157 & 0.187 & 0.215 & 0.244 & 0.274 & 0.305 & 0.337 & 0.373 \\
\hline 9 & 0.000 & 0.077 & 0.112 & 0.142 & 0.169 & 0.195 & 0.221 & 0.247 & 0.275 & 0.305 & 0.337 \\
\hline 10 & 0.000 & 0.071 & 0.103 & 0.129 & 0.154 & 0.178 & 0.202 & 0.226 & 0.251 & 0.279 & 0.308 \\
\hline 11 & 0.000 & 0.065 & 0.095 & 0.120 & 0.143 & 0.165 & 0.187 & 0.209 & 0.233 & 0.258 & 0.285 \\
\hline 12 & 0.000 & 0.061 & 0.089 & 0.112 & 0.133 & 0.154 & 0.174 & 0.195 & 0.217 & 0.241 & 0.266 \\
\hline 13 & 0.000 & 0.057 & 0.083 & 0.105 & 0.125 & 0.144 & 0.163 & 0.183 & 0.203 & 0.225 & 0.249 \\
\hline 14 & 0.000 & 0.054 & 0.078 & 0.099 & 0.118 & 0.136 & 0.154 & 0.172 & 0.192 & 0.213 & 0.235 \\
\hline 15 & 0.000 & 0.051 & 0.074 & 0.094 & 0.112 & 0.129 & 0.146 & 0.164 & 0.182 & 0.202 & 0.223 \\
\hline 16 & 0.000 & 0.049 & 0.071 & 0.089 & 0.106 & 0.122 & 0.139 & 0.156 & 0.173 & 0.192 & 0.212 \\
\hline 17 & 0.000 & 0.047 & 0.068 & 0.085 & 0.102 & 0.117 & 0.133 & 0.149 & 0.166 & 0.184 & 0.203 \\
\hline 18 & 0.000 & 0.045 & 0.065 & 0.081 & 0.097 & 0.112 & 0.127 & 0.142 & 0.158 & 0.175 & 0.194 \\
\hline 19 & 0.000 & 0.043 & 0.062 & 0.079 & 0.094 & 0.108 & 0.122 & 0.137 & 0.153 & 0.169 & 0.187 \\
\hline 20 & 0.000 & 0.041 & 0.060 & 0.076 & 0.090 & 0.104 & 0.118 & 0.132 & 0.147 & 0.163 & 0.180 \\
\hline
\end{tabular}


where $d_{2}^{Q}$ is a constant whose value depends on the sample size $n$ (see Table 1). The standard deviation, $\sigma_{X}$ is estimated by

$$
\hat{\sigma}_{X}=\frac{\overline{\mathrm{IQR}}}{d_{2}^{Q}}
$$

where $\overline{\mathrm{IQR}}$ is the average of the subgroup interquartile ranges.

Assuming that all the observed data are independent from one sample to another, then as $t$ increases,

$$
\operatorname{Var}\left(\hat{W}_{t}\right)=\operatorname{Var}(\bar{X}) \frac{\alpha}{2-\alpha}
$$

and

$$
\sigma_{\hat{W}_{t}}=\frac{\sigma_{X}}{\sqrt{n}} \sqrt{\frac{\alpha}{2-\alpha}} .
$$

From equation (6), by using the average interquartile range to estimate the standard deviation of $\hat{W}_{t}, \hat{\sigma}_{\hat{W}_{t}}$, the following is obtained

$$
\hat{\sigma}_{\hat{w}_{t}}=\frac{\overline{\mathrm{IQR}}}{d_{2}^{Q} \sqrt{n}} \sqrt{\frac{\alpha}{2-\alpha}} .
$$

Thus, the limits of the EWMASMQ chart are

Center Line $=\hat{W}_{0}=\overline{\bar{X}}$

Control Limits $=\hat{W}_{0} \pm 3 \hat{\sigma}_{\hat{W}_{i}}$

$$
=\overline{\bar{X}} \pm 3 \frac{\overline{\mathrm{IQR}}}{d_{2}^{Q} \sqrt{n}} \sqrt{\frac{\alpha}{2-\alpha}}
$$

If

$$
G_{1}=\frac{3}{d_{2}^{Q} \sqrt{n}} \sqrt{\frac{\alpha}{2-\alpha}}
$$

then the control limits calculated based on equation (11) are

$$
\mathrm{UCL}_{\mathrm{SMQ}}=\overline{\bar{X}}+G_{1} \overline{\mathrm{IQR}}
$$

\begin{tabular}{|c|c|c|c|c|c|c|c|c|c|c|c|}
\hline \multirow{2}{*}{$\begin{array}{l}\text { Sample } \\
\text { Size, } n\end{array}$} & \multicolumn{11}{|c|}{ Value of $\alpha$} \\
\hline & 0 & 0.1 & 0.2 & 0.3 & 0.4 & 0.5 & 0.6 & 0.7 & 0.8 & 0.9 & 1 \\
\hline 2 & 0.0000 & 0.4313 & 0.6266 & 0.7897 & 0.9400 & 1.0854 & 1.2307 & 1.3795 & 1.5350 & 1.7005 & 1.8799 \\
\hline 3 & 0.0000 & 0.2348 & 0.3411 & 0.4299 & 0.5117 & 0.5908 & 0.6699 & 0.7509 & 0.8355 & 0.9256 & 1.0233 \\
\hline 4 & 0.0000 & 0.5793 & 0.8418 & 1.0608 & 1.2626 & 1.4580 & 1.6532 & 1.8530 & 2.0619 & 2.2842 & 2.5253 \\
\hline 5 & 0.0000 & 0.3109 & 0.4517 & 0.5693 & 0.6776 & 0.7824 & 0.8872 & 0.9944 & 1.1065 & 1.2258 & 1.3552 \\
\hline 6 & 0.0000 & 0.2189 & 0.3181 & 0.4009 & 0.4771 & 0.5509 & 0.6247 & 0.7002 & 0.7791 & 0.8631 & 0.9542 \\
\hline 7 & 0.0000 & 0.1717 & 0.2495 & 0.3145 & 0.3743 & 0.4322 & 0.4901 & 0.5493 & 0.6112 & 0.6771 & 0.7486 \\
\hline 8 & 0.0000 & 0.2573 & 0.3739 & 0.4712 & 0.5608 & 0.6476 & 0.7343 & 0.8231 & 0.9158 & 1.0146 & 1.1217 \\
\hline 9 & 0.0000 & 0.2006 & 0.2914 & 0.3672 & 0.4371 & 0.5047 & 0.5723 & 0.6415 & 0.7138 & 0.7907 & 0.8742 \\
\hline 10 & 0.0000 & 0.1659 & 0.2410 & 0.3037 & 0.3615 & 0.4174 & 0.4733 & 0.5306 & 0.5903 & 0.6540 & 0.7230 \\
\hline 11 & 0.0000 & 0.1424 & 0.2068 & 0.2607 & 0.3103 & 0.3583 & 0.4062 & 0.4553 & 0.5067 & 0.5613 & 0.6205 \\
\hline 12 & 0.0000 & 0.1850 & 0.2689 & 0.3388 & 0.4033 & 0.4657 & 0.5280 & 0.5919 & 0.6586 & 0.7296 & 0.8066 \\
\hline 13 & 0.0000 & 0.1583 & 0.2300 & 0.2899 & 0.3450 & 0.3984 & 0.4518 & 0.5064 & 0.5635 & 0.6242 & 0.6901 \\
\hline 14 & 0.0000 & 0.1390 & 0.2019 & 0.2545 & 0.3029 & 0.3498 & 0.3966 & 0.4445 & 0.4946 & 0.5480 & 0.6058 \\
\hline 15 & 0.0000 & 0.1243 & 0.1806 & 0.2276 & 0.2709 & 0.3128 & 0.3547 & 0.3975 & 0.4423 & 0.4900 & 0.5418 \\
\hline 16 & 0.0000 & 0.1509 & 0.2193 & 0.2764 & 0.3289 & 0.3798 & 0.4307 & 0.4828 & 0.5372 & 0.5951 & 0.6579 \\
\hline 17 & 0.0000 & 0.1347 & 0.1958 & 0.2467 & 0.2937 & 0.3391 & 0.3845 & 0.4310 & 0.4795 & 0.5312 & 0.5873 \\
\hline 18 & 0.0000 & 0.1223 & 0.1776 & 0.2239 & 0.2665 & 0.3077 & 0.3489 & 0.3910 & 0.4351 & 0.4820 & 0.5329 \\
\hline 19 & 0.0000 & 0.1117 & 0.1623 & 0.2046 & 0.2435 & 0.2812 & 0.3188 & 0.3574 & 0.3976 & 0.4405 & 0.4870 \\
\hline 20 & 0.0000 & 0.1304 & 0.1894 & 0.2387 & 0.2841 & 0.3281 & 0.3720 & 0.4169 & 0.4639 & 0.5140 & 0.5682 \\
\hline
\end{tabular}

and

$$
\mathrm{LCL}_{\mathrm{SMQ}}=\overline{\bar{X}}-G_{1} \overline{\mathrm{IQR}} \text {. }
$$

Values of $G_{1}$ for different sample sizes, $n$, and smoothing constants, $\alpha$, are listed in Table 3. 
Comparison Of Performances

The performance of the EWMASMQ chart is compared to that of the EWMASM by performing a Monte-Carlo simulation. The following four different conditions are considered for the two control charts for the process mean:

(i) The In-control situation where the data are all standard normal random variables.

(ii) The Outliers situation where the data are a mixture of $95 \%$ standard normal and 5\% data with five times the standard deviation. These outliers might represent an episodic phenomenon resulting from a sporadic special cause that control charts should detect.

(iii) The Special Cause situation where there is an additional $N(\delta, 1)$ source of variability added to the subgroup means. Here, $\delta=\frac{\mu-\mu_{0}}{\sigma_{0}}$ where $\mu_{0}=0$ and $\sigma_{0}=1$ represent the nominal mean and standard deviation respectively while $\mu$ is the off-target mean. The values of $\delta \in\{0,0.25,0.5,0.75,1,1.5,2,2.5$, $3,4\}$ are considered. The control charts should detect this special cause.

(iv) The Outliers and Special Cause situation which consists of the data that are a mixture of $95 \%$ standard normal and $5 \%$ data with five times the standard deviation together with an additional component of variation, $N(\delta, 1)$ added to the subgroup means.
Simulation studies based on the above four conditions are conducted using SAS version 8. Repeatedly, $m=10$ and 20 subgroups of size $n=5$ observations each are generated, control limits computed and the number of subgroups that fall outside the control limits are calculated. This procedure is repeated 10000 times for a total of $10000 \times m$ subgroups. The proportions of out-of-control subgroups (based on $10000 \times$ $m$ subgroups) are computed for the four different conditions and two different charting methods.

The results for the EWMASM chart are displayed in Tables 4 and 5 for $m=10$ and 20 respectively. Similarly, Tables 6 and 7 give the results of the EWMASMQ chart for $m=10$ and 20 respectively. An ideal procedure is the chart which gives a higher proportion for detecting out-of-control signals for the three conditions of Outliers, Special Cause and Outliers and Special Cause and a lower signal proportion for the Incontrol situation.

A comparison of the results in Tables 4 and 6 for $m=10$ show that for fixed values of $\alpha$ and $\delta$, the out-of-control proportions of the EWMASMQ chart are higher than the corresponding values of the EWMASM chart for the two out-of-control conditions of Outliers and Outliers and Special Cause. For example, when $\alpha=0.5$, the out-of-control proportion in Table 6 is 0.0242 while that in Table 4 is much lower at only 0.0047 for the Outliers condition. For the Outliers and Special Cause condition, the proportions of out-of-control in Table 4 when $\alpha=0.5$ are $\{0.0194,0.0388, \ldots, 0.9976\}$ while the corresponding proportions in Table 6 are $\{0.0554,0.0878, \ldots, 0.9992\}$ where the values of the former are all lower than that of the latter for $\delta \in\{0,0.25, \ldots, 4\}$. 
This shows that the EWMASMQ chart is more sensitive to out-of-control conditions when outliers are present in the data. The limits computed from the estimate of the interquartile ranges for the EWMASMQ chart are less influenced by outliers, compared to that of the EWMASM chart whose limits are computed based on the sample ranges. Thus, the EWMASMQ chart is more robust than the
EWMASM and the former is a better alternative in the detection of a special cause when outliers are present. The EWMASMQ chart is also superior to the EWMASM when only outliers are present. The results in both Tables 4 and 6 indicate that for the Special Cause condition, the EWMASMQ chart is superior to the EWMASM for smaller values of $\delta$ and that both charts have comparable performances for larger values of $\delta$.

Table 4. Proportions of Out-of-Control for the EWMASM Chart Under Four Different Conditions based on $m=10$ and $n=5$

\begin{tabular}{|c|c|c|c|c|c|c|c|c|c|c|}
\hline & \multicolumn{9}{|c|}{$\begin{array}{c}\text { EWMASM } \\
\alpha\end{array}$} \\
\hline & & $\begin{array}{l}0.1 \\
\end{array}$ & 0.2 & 0.3 & $\begin{array}{l}0.4 \\
\end{array}$ & 0.5 & 0.6 & 0.7 & 0.8 & 0.9 \\
\hline \multicolumn{2}{|c|}{ In-Control } & 0.0000 & 0.0001 & 0.0003 & 0.0005 & 0.0009 & 0.0013 & 0.0017 & 0.0022 & 0.0025 \\
\hline \multicolumn{2}{|l|}{ Outliers } & 0.0000 & 0.0002 & 0.0009 & 0.0023 & 0.0047 & 0.0066 & 0.0093 & 0.0107 & 0.0126 \\
\hline & $\delta$ & & & & & & & & & \\
\hline \multirow{12}{*}{ 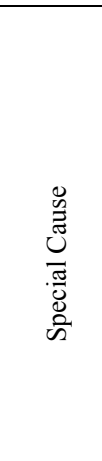 } & 0 & 0.0030 & 0.0106 & 0.0168 & 0.0214 & 0.0256 & 0.0286 & 0.0311 & 0.0327 & 0.0338 \\
\hline & 0.25 & 0.0377 & 0.0601 & 0.0638 & 0.0618 & 0.0600 & 0.0568 & 0.0550 & 0.0528 & 0.0504 \\
\hline & 0.5 & 0.2395 & 0.2786 & 0.2502 & 0.2114 & 0.1816 & 0.1546 & 0.1353 & 0.1187 & 0.1056 \\
\hline & 0.75 & 0.5162 & 0.5703 & 0.5337 & 0.4633 & 0.3946 & 0.3317 & 0.2829 & 0.2399 & 0.2045 \\
\hline & 1 & 0.6813 & 0.7482 & 0.7484 & 0.7035 & 0.6341 & 0.5529 & 0.4772 & 0.4090 & 0.3483 \\
\hline & 1.5 & 0.8246 & 0.8803 & 0.9051 & 0.9150 & 0.9097 & 0.8809 & 0.8296 & 0.7606 & 0.6802 \\
\hline & 2 & 0.8858 & 0.9323 & 0.9559 & 0.9684 & 0.9750 & 0.9760 & 0.9684 & 0.9478 & 0.9077 \\
\hline & 2.5 & 0.9181 & 0.9661 & 0.9839 & 0.9899 & 0.9929 & 0.9942 & 0.9948 & 0.9929 & 0.9847 \\
\hline & 3 & 0.9469 & 0.9880 & 0.9953 & 0.9977 & 0.9985 & 0.9990 & 0.9992 & 0.9993 & 0.9986 \\
\hline & 4 & 0.9904 & 0.9995 & 1.0000 & 1.0000 & 1.0000 & 1.0000 & 1.0000 & 1.0000 & 1.0000 \\
\hline & 0 & 0.0013 & 0.0056 & 0.0108 & 0.0154 & 0.0194 & 0.0234 & 0.0262 & 0.0287 & 0.0303 \\
\hline & 0.25 & 0.0159 & 0.0296 & 0.0366 & 0.0381 & 0.0388 & 0.0399 & 0.0397 & 0.0401 & 0.0394 \\
\hline \multirow{8}{*}{ 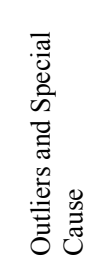 } & 0.5 & 0.1241 & 0.1568 & 0.1457 & 0.1255 & 0.1097 & 0.0961 & 0.0853 & 0.0773 & 0.0700 \\
\hline & 0.75 & 0.3492 & 0.3908 & 0.3535 & 0.2971 & 0.2480 & 0.2071 & 0.1749 & 0.1490 & 0.1268 \\
\hline & 1 & 0.5464 & 0.6025 & 0.5726 & 0.5070 & 0.4363 & 0.3643 & 0.3064 & 0.2574 & 0.2176 \\
\hline & 1.5 & 0.7440 & 0.8079 & 0.8214 & 0.8027 & 0.7630 & 0.7000 & 0.6265 & 0.5484 & 0.4728 \\
\hline & 2 & 0.8309 & 0.8847 & 0.9084 & 0.9169 & 0.9114 & 0.8881 & 0.8511 & 0.7953 & 0.7276 \\
\hline & 2.5 & 0.8792 & 0.9270 & 0.9499 & 0.9609 & 0.9644 & 0.9605 & 0.9487 & 0.9243 & 0.8859 \\
\hline & 3 & 0.9091 & 0.9553 & 0.9739 & 0.9819 & 0.9857 & 0.9857 & 0.9824 & 0.9728 & 0.9569 \\
\hline & 4 & 0.9542 & 0.9872 & 0.9944 & 0.9967 & 0.9976 & 0.9981 & 0.9979 & 0.9964 & 0.9933 \\
\hline
\end{tabular}


Table 5. Proportions of Out-of-Control for the EWMASM Chart Under Four Different Conditions based on $m=20$ and $n=5$

\begin{tabular}{|c|c|c|c|c|c|c|c|c|c|c|}
\hline & \multicolumn{9}{|c|}{$\begin{array}{c}\text { EWMASM } \\
\alpha \\
\end{array}$} \\
\hline & & 0.1 & 0.2 & 0.3 & 0.4 & 0.5 & 0.6 & 0.7 & 0.8 & 0.9 \\
\hline \multicolumn{2}{|c|}{ In-Control } & 0.0000 & 0.0003 & 0.0007 & 0.0012 & 0.0017 & 0.0020 & 0.0024 & 0.0026 & 0.0029 \\
\hline \multicolumn{2}{|l|}{ Outliers } & 0.0001 & 0.0017 & 0.0048 & 0.0080 & 0.0113 & 0.0140 & 0.0160 & 0.0180 & 0.0191 \\
\hline & $\delta$ & & & & & & & & & \\
\hline \multirow{12}{*}{ 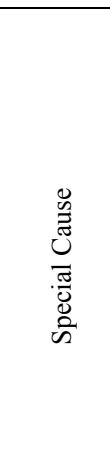 } & 0 & 0.0079 & 0.0170 & 0.0228 & 0.0258 & 0.0287 & 0.0306 & 0.0320 & 0.0330 & 0.0338 \\
\hline & 0.25 & 0.1162 & 0.1045 & 0.0879 & 0.0753 & 0.0678 & 0.0612 & 0.0574 & 0.0538 & 0.0507 \\
\hline & 0.5 & 0.5068 & 0.4269 & 0.3252 & 0.2510 & 0.2035 & 0.1677 & 0.1425 & 0.1222 & 0.1064 \\
\hline & 0.75 & 0.7492 & 0.7439 & 0.6492 & 0.5316 & 0.4343 & 0.3545 & 0.2948 & 0.2463 & 0.2078 \\
\hline & 1 & 0.8381 & 0.8695 & 0.8503 & 0.7784 & 0.6844 & 0.5848 & 0.4961 & 0.4182 & 0.3514 \\
\hline & 1.5 & 0.9114 & 0.9392 & 0.9516 & 0.9547 & 0.9427 & 0.9071 & 0.8477 & 0.7712 & 0.6855 \\
\hline & 2 & 0.9424 & 0.9658 & 0.9776 & 0.9836 & 0.9870 & 0.9861 & 0.9772 & 0.9533 & 0.9116 \\
\hline & 2.5 & 0.9588 & 0.9831 & 0.9918 & 0.9951 & 0.9965 & 0.9973 & 0.9974 & 0.9951 & 0.9867 \\
\hline & 3 & 0.9728 & 0.9943 & 0.9979 & 0.9991 & 0.9995 & 0.9996 & 0.9997 & 0.9996 & 0.9990 \\
\hline & 4 & 0.9958 & 0.9998 & 1.0000 & 1.0000 & 1.0000 & 1.0000 & 1.0000 & 1.0000 & 1.0000 \\
\hline & 0 & 0.0039 & 0.0117 & 0.0183 & 0.0227 & 0.0268 & 0.0293 & 0.0318 & 0.0330 & 0.0338 \\
\hline & 0.25 & 0.0545 & 0.0582 & 0.0524 & 0.0488 & 0.0465 & 0.0446 & 0.0442 & 0.0430 & 0.0420 \\
\hline \multirow{8}{*}{ 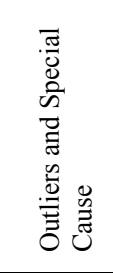 } & 0.5 & 0.3232 & 0.2577 & 0.1893 & 0.1464 & 0.1188 & 0.0997 & 0.0867 & 0.0767 & 0.0684 \\
\hline & 0.75 & 0.6173 & 0.5575 & 0.4401 & 0.3358 & 0.2653 & 0.2095 & 0.1731 & 0.1443 & 0.1225 \\
\hline & 1 & 0.7609 & 0.7622 & 0.6824 & 0.5685 & 0.4679 & 0.3764 & 0.3086 & 0.2497 & 0.2067 \\
\hline & 1.5 & 0.8699 & 0.9021 & 0.9029 & 0.8721 & 0.8161 & 0.7331 & 0.6411 & 0.5487 & 0.4626 \\
\hline & 2 & 0.9154 & 0.9414 & 0.9537 & 0.9555 & 0.9462 & 0.9196 & 0.8770 & 0.8109 & 0.7322 \\
\hline & 2.5 & 0.9392 & 0.9629 & 0.9749 & 0.9808 & 0.9815 & 0.9772 & 0.9635 & 0.9400 & 0.8995 \\
\hline & 3 & 0.9534 & 0.9772 & 0.9878 & 0.9921 & 0.9936 & 0.9930 & 0.9893 & 0.9810 & 0.9664 \\
\hline & 4 & 0.9763 & 0.9947 & 0.9979 & 0.9988 & 0.9991 & 0.9993 & 0.9990 & 0.9980 & 0.9954 \\
\hline
\end{tabular}

Table 6. Proportions of Out-of-Control for the EWMASMQ Chart Under Four Different Conditions based on $m=10$ and $n=5$

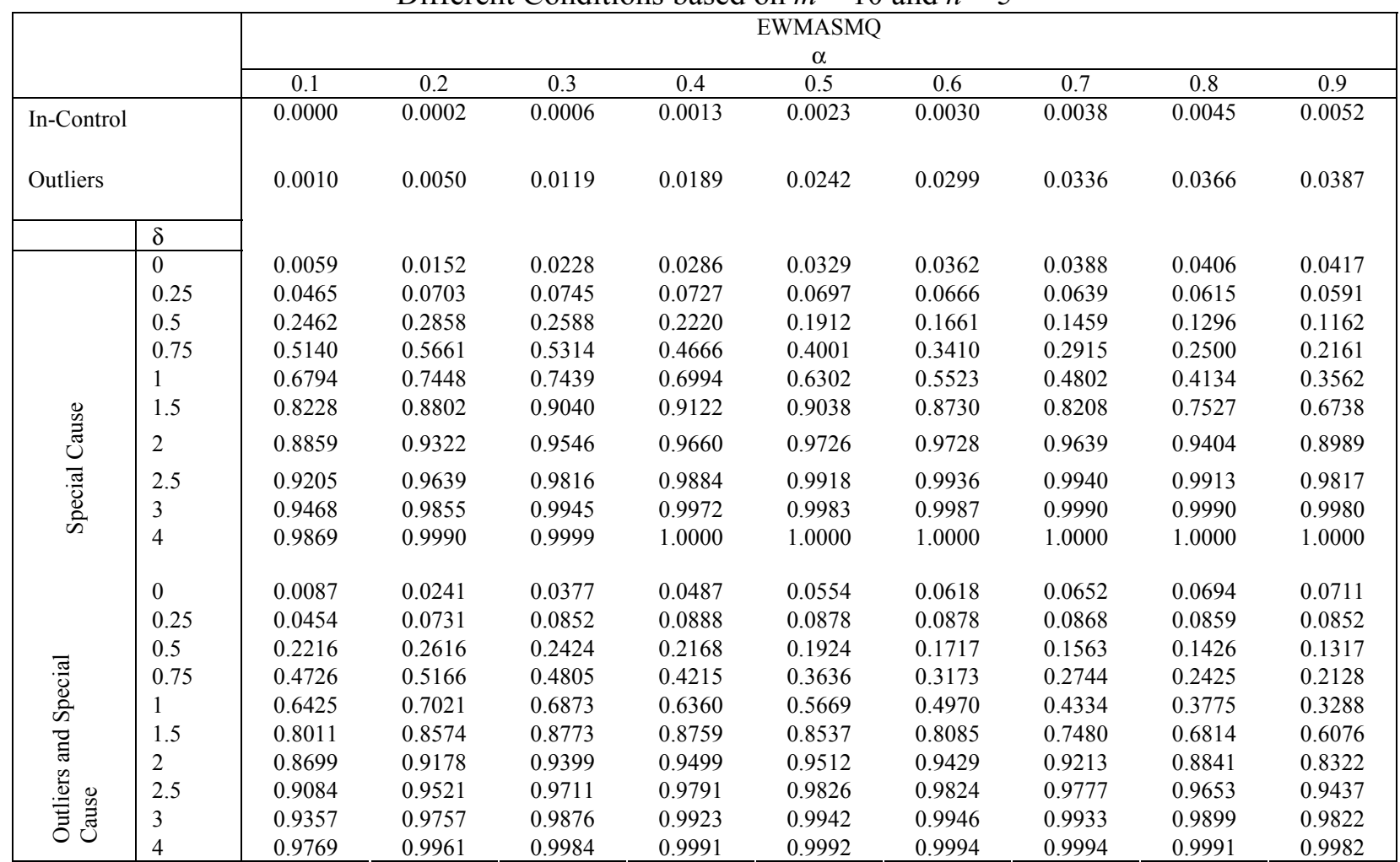


It is shown by the results in Tables 4 and 6 that the EWMASM chart has lower false alarm (Type-I error) rates than the EWMASMQ chart. However, it should be noted that the superiority of the EWMASMQ chart in comparison to the EWMASM chart for the two out-of-control conditions of Outliers and Outliers and Special Cause far outweigh its deficiency in terms of a higher rate of false alarm. For example, if $\alpha=$ 0.3 , the proportion of false alarm of the EWMASMQ chart is twice that of the EWMASM but the former is thirteen times more efficient in detecting outliers compared to the latter. Because a comparison between Tables 5 and 7 shows similar trend, it is suffice to discuss and draw conclusions based on the above comparisons.

Note that the evaluation of the performances of the control charts discussed in this section are made based on the proportions of subgroup points falling outside the control limits and are not based on the average run length (ARL) values because the charts' parameters are estimated from the subgroup data, i.e., the nominal values of these parameters are assumed to be unknown.

Table 7. Proportions of Out-of-Control for the EWMASMQ Chart Under Four Different Conditions based on $m=20$ and $n=5$

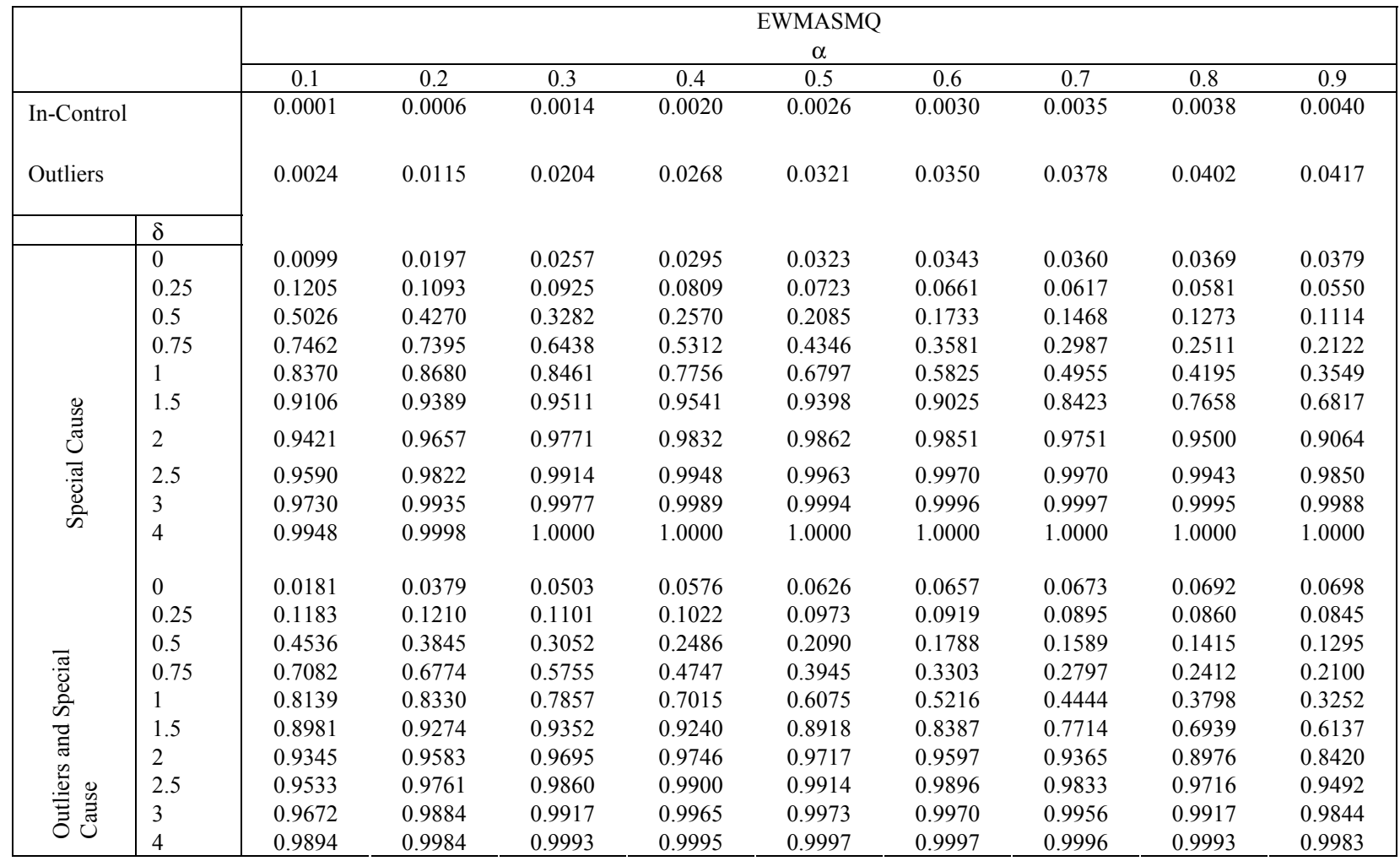


An Example of Application

An example will be given to illustrate how the EWMASMQ chart is put to work in a real situation. The EWMASM chart is also constructed so that a comparison between the two approaches can be made. This example is based on the data from Wadsworth, Stephens and Godfrey (1986) and concerns the melt index of an extrusion grade polyethylene compound. As part of a study of the process, 20 subgroups of four each are taken. Table 8 gives the data of this process.
The limits of the EWMASM chart are computed using equations (2a) and (2b) while that of the EWMASMQ chart are calculated from equations (13a) and (13b). Figure 1 shows the EWMASM and EWMASMQ charts together with their respective control limits. The limits of the EWMASM chart are represented by UCL/LCL and those of the EWMASMQ chart by $\mathrm{UCL}_{\mathrm{SMQ}} / \mathrm{LCL}_{\mathrm{SMQ}}$.

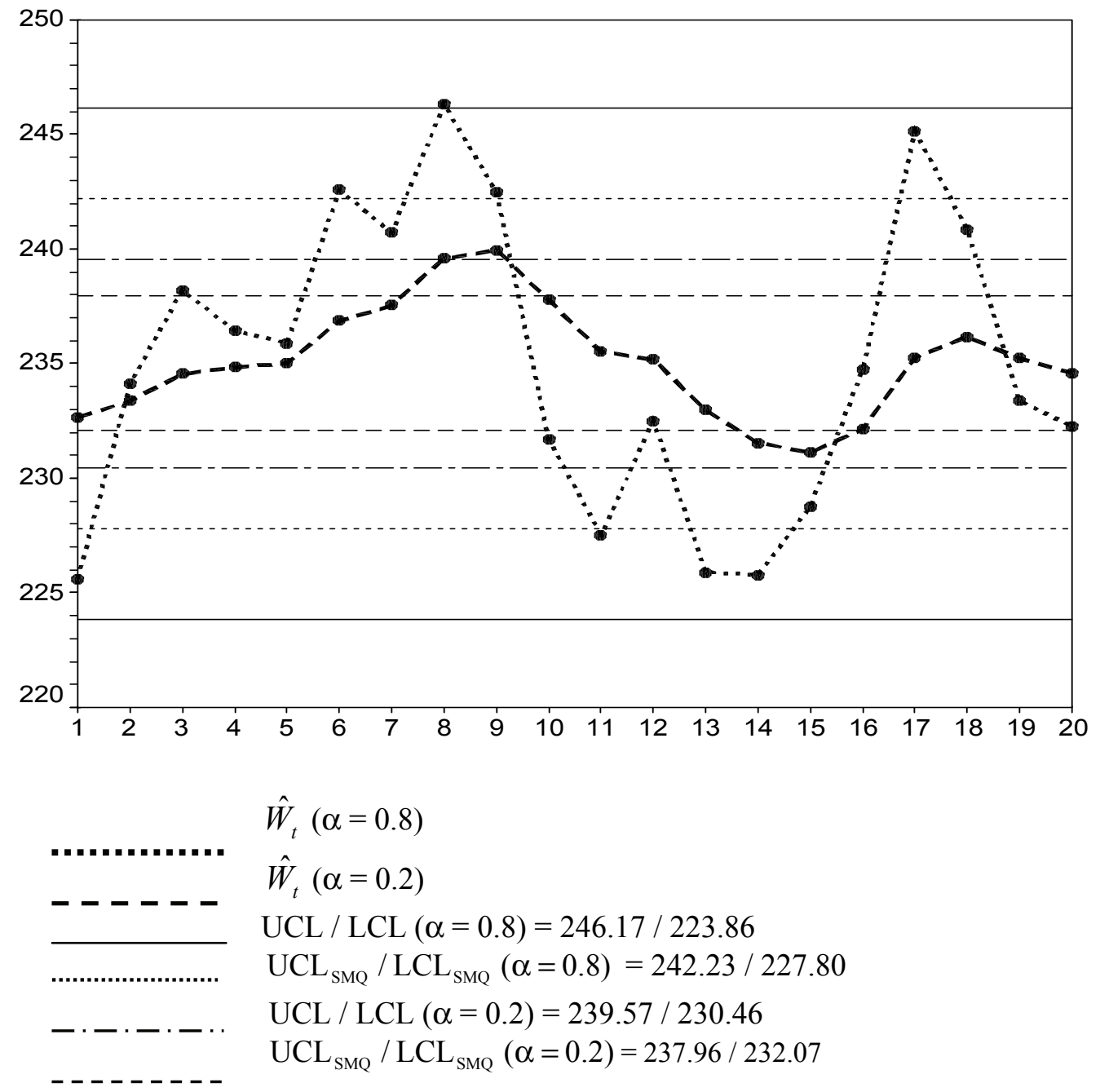

Figure 1. The EWMASM and EWMASMQ Charts for Melt Index Data 
The EWMASM chart with $\alpha=0.2$ detects out-of-control points at subgroups 8 and 9 while the corresponding EWMASMQ chart signals at subgroups 8, 9, 14 and 15. The EWMASMQ chart detects two additional out-ofcontrol points (i.e., subgroups 14 and 15) besides the two points at subgroups 8 and 9 which are also detected by the EWMASM chart. From Figure 1 for $\alpha=0.2$, a shift is observed that is gradually increasing between subgroups 1 and 9 followed by a shift which is gradually decreasing until subgroup 15. Here, both the EWMASM and EWMASMQ charts have successfully detected the upward shift but only the EWMASMQ chart managed to detect the downward shift. The presence of sample ranges with large values such as those in subgroups 3 , 4, 6 and 8 cause the average sample range, $\bar{R}$ to be overestimated, hence widening the limits of the EWMASM chart so that the chart is less sensitive in detecting shifts in the mean. On the contrary, the EWMASMQ chart does not face this problem since its limits are computed based on the average sample interquartile range (IQR).

The EWMASM and EWMASMQ charts with $\alpha=0.8$ give more weight to the current sample average, $\bar{X}_{t}$ compared to the charts with $\alpha=0.2$. Thus, a weighting constant of $\alpha=0.8$ makes the two charts more sensitive to single subgroup averages with big or small values. Out-of-control points are detected at subgroups $1,6,8,9,11,13,14$ and 17 by the EWMASMQ chart and at only subgroup 8 by the EWMASM chart. From the sample averages, $\bar{X}_{t}$ in Table 8 , we notice that the values for subgroups $6,8,9$ and 17 are somewhat bigger while those for subgroups 1, 11, 13 and 14 are somewhat smaller than the other sample averages. Investigations need to be carried out to search for assignable causes before these subgroups are classified as out-of-control points. It should be noted that the EWMASM chart with $\alpha=0.8$ fails to detect any subgroup average that plots below the LCL.

Table 8. Subgroups of Melt Index Measurements and the Computed Sample Means, Sample Ranges, Sample Interquartile Ranges and $\hat{W}_{t}$ Statistics

\begin{tabular}{|c|c|c|c|c|c|c|c|c|c|}
\hline \multirow{2}{*}{$\begin{array}{c}\text { Sub. No., } \\
t\end{array}$} & \multicolumn{4}{|c|}{ Observations } & \multirow{2}{*}{$\bar{X}_{t}$} & \multirow{2}{*}{$R_{t}$} & \multirow{2}{*}{$\mathrm{IQR}_{t}$} & \multicolumn{2}{|c|}{$\hat{W}_{t}$} \\
\hline & $X_{1}$ & $X_{2}$ & $X_{3}$ & $X_{4}$ & & & & $\alpha=0.2$ & $\alpha=0.8$ \\
\hline 1 & 218 & 224 & 220 & 231 & 223.25 & 13 & 4 & 232.66 & 225.60 \\
\hline 2 & 228 & 236 & 247 & 234 & 236.25 & 19 & 2 & 233.38 & 234.12 \\
\hline 3 & 280 & 228 & 228 & 221 & 239.25 & 59 & 0 & 234.55 & 238.22 \\
\hline 4 & 210 & 249 & 241 & 246 & 236 & 39 & 5 & 234.84 & 236.45 \\
\hline 5 & 243 & 240 & 230 & 230 & 235.75 & 13 & 10 & 235.02 & 235.89 \\
\hline 6 & 225 & 250 & 258 & 244 & 244.25 & 33 & 6 & 236.87 & 242.58 \\
\hline 7 & 240 & 238 & 240 & 243 & 240.25 & 5 & 0 & 237.55 & 240.72 \\
\hline 8 & 244 & 248 & 265 & 234 & 247.75 & 31 & 4 & 239.59 & 246.34 \\
\hline 9 & 238 & 233 & 252 & 243 & 241.5 & 19 & 5 & 239.97 & 242.47 \\
\hline 10 & 228 & 238 & 220 & 230 & 229 & 18 & 2 & 237.78 & 231.69 \\
\hline 11 & 218 & 232 & 230 & 226 & 226.5 & 14 & 4 & 235.52 & 227.54 \\
\hline 12 & 226 & 231 & 236 & 242 & 233.75 & 16 & 5 & 235.17 & 232.51 \\
\hline 13 & 224 & 221 & 230 & 222 & 224.25 & 9 & 2 & 232.98 & 225.90 \\
\hline 14 & 230 & 220 & 227 & 226 & 225.75 & 10 & 1 & 231.54 & 225.78 \\
\hline 15 & 224 & 228 & 226 & 240 & 229.5 & 16 & 2 & 231.13 & 228.76 \\
\hline 16 & 232 & 240 & 241 & 232 & 236.25 & 9 & 8 & 232.15 & 234.75 \\
\hline 17 & 243 & 250 & 248 & 250 & 247.75 & 7 & 2 & 235.27 & 245.15 \\
\hline 18 & 247 & 238 & 244 & 230 & 239.75 & 17 & 6 & 236.17 & 240.83 \\
\hline 19 & 224 & 228 & 228 & 246 & 231.5 & 22 & 0 & 235.23 & 233.37 \\
\hline 20 & 236 & 230 & 230 & 232 & 232 & 6 & 2 & 234.59 & 232.27 \\
\hline & & & & & $\begin{array}{c}\overline{\bar{X}}= \\
235.0125\end{array}$ & $\begin{array}{l}\bar{R}= \\
18.75\end{array}$ & $\begin{array}{c}\mathrm{IQR}= \\
3.5\end{array}$ & & \\
\hline
\end{tabular}




\section{Conclusion}

This article discusses a new robust EWMA control chart for the sample mean which is referred to as the EWMASMQ chart. It is shown by simulation that the EWMASMQ chart is a superior alternative to the EWMASM chart when one is concern with the presence of outliers. Generally, the new EWMASMQ chart allows easier detection of outliers in the subgroups and is also more sensitive to other forms of out-of-control behavior when outliers are present. An example is given to show how the EWMASMQ chart works in a real situation. This example also illustrates the superiority of the EWMASMQ chart to the EWMASM chart, hence making the EWMASMQ as an attractive alternative to quality control practitioners.

\section{References}

Borror, C. M., Champ, C. W., \& Rigdon, S. E. (1998). Poisson EWMA control charts. Journal of Quality Technology, 30, 352361.

Crowder, S. V. (1987). A simple method for studying run-length distributions of exponentially weighted moving average charts. Technometrics, 29, 401-407.

Crowder, S. V. (1989). Design of exponentially weighted moving average schemes. Journal of Quality Technology, 21, 155-162.

Gan, F. F. (1990). Monitoring Poisson observations using modified exponentially weighted moving average control charts. Communications in Statistics - Simulation and Computation, 103-124.
Lucas, J. M. \& Saccucci, M. S. (1990). Exponentially weighted moving average control schemes: properties and enhancements. Technometrics, 32, 1-12.

MacGregor, J. F. \& Harris, T. J. (1993). The exponentially weighted moving variance. Journal of Quality Technology, 25, 106-118.

$\mathrm{Ng}$, C. H. \& Case, K. E. (1989). Development and evaluation of control charts using exponentially weighted moving averages. Journal of Quality Technology, 21, 242-250.

Rhoads, T. R., Montgomery, D. C., \& Mastrangelo, C. M. (1996). A fast initial response scheme for the exponentially weighted moving average control chart. Quality Engineering, 9, 317-327.

Roberts, S. W. (1959). Control chart based on geometric moving averages. Technometrics, 1, 239-250.

Rocke, D. M. (1989). Robust control charts. Technometrics, 31, 173-184.

Rocke, D. M. (1992). $\bar{X}_{\mathrm{Q}}$ and $R_{\mathrm{Q}}$ charts: Robust control charts. The Statistician, 41, 97-104.

Somerville, S. E., Montgomery, D. C., \& Runger, G. C. (2002). Filtering and smoothing methods for mixed particle count distributions. International Journal of Production Research, 40, 2991-3013.

Sweet, A. L. (1986). Control chart using coupled EWMA. IIE Transactions, 18, 26-33.

Wadsworth, H. M., Stephens, K. S., \& Godfrey, A. B. (1986). Modern methods for quality control and improvement. New York, N.Y.: John Wiley \& Sons. 